\title{
ESTOQUE E DINÂMICA DE BIOMASSA ARBÓREA EM FLORESTA OMBRÓFILA DENSA NA FLONA TAPAJÓS: AMAZÔNIA ORIENTAL
}

\author{
STOCKS AND DYNAMICS OF TREE BIOMASS IN TROPICAL RAIN FOREST IN THE TAPAJOS \\ NATIONAL FOREST, EASTERN AMAZONIA
}

Fabio Guerra Santos ${ }^{1}$ Plinio Barbosa de Camargo² Raimundo Cosme de Oliveira Junior ${ }^{3}$

\section{RESUMO}

A análise das oscilações dos estoques de biomassa em florestas tropicais permite descrever o comportamento desses ecossistemas diante de alterações naturais ou antropogênicas. O objetivo deste trabalho foi avaliar o estoque e a dinâmica de biomassa no período (2007-2010) em Floresta Ombrófila Densa de duas áreas de pesquisa na FLONA - Tapajós: Amazônia oriental, denominadas Área 1 e Área 2, distantes aproximadamente $45 \mathrm{~km}$ uma da outra. A biomassa foi estimada através de equação alométrica e medidas de DAP, proveniente de 12 parcelas permanentes, sendo $6 \mathrm{em}$ cada área. Os resultados mostraram que houve diferença significativa no estoque médio anual de biomassa entre a Área $1(293,19 \pm 27,74 ; 298,21$ $\pm 31,73 ; 299,60 \pm 29,46 ; 298,11 \pm 29,40 \mathrm{Mg}$ ha $\left.^{-1}\right)$ e a Área $2(254,35 \pm 69,61 ; 259,10 \pm 70,05 ; 261,00 \pm$ 69,$\left.43 ; 248,92 \pm 61,78 \mathrm{Mg} \cdot \mathrm{ha}^{-1}\right)$. Notou-se variação anual no estoque de biomassa em intervalos de medições superiores a um ano, devido às oscilações na taxa de mortalidade, recrutamento e incremento, além da área basal e abundância dos indivíduos em cada área. A maior concentração de indivíduos ocorreu nas classes diamétricas $10 \leq \mathrm{DAP} \leq 30 \mathrm{~cm}$, porém, verificou-se que os indivíduos com DAP $\geq 60 \mathrm{~cm}$ em concentração menor estocam biomassa equivalente à da classe diamétrica $10 \leq \mathrm{DAP} \leq 30 \mathrm{~cm}$. Concluiuse que as oscilações na taxa de mortalidade, recrutamento e incremento, além da abundância e área basal nestes locais afetaram os estoques de biomassa.

Palavras-chave: método indireto; distribuição diamétrica; abundância.

\begin{abstract}
The analysis of the oscillations of biomass stocks in tropical forests allows us to describe the behavior of these ecosystems in the face of natural or anthropogenic changes. The objective of this study was to evaluate the inventory and dynamics of biomass in the period (2007-2010) in dense rain forest in two areas of research in the National Forest - Tapajos, eastern Amazonia, called Area 1 and Area 2, $45 \mathrm{~km}$ distant from each other. The biomass was estimated using allometric equation and DAP, from 12 permanent plots, with six in each area. The results showed a significant difference in annual average stock biomass between Area $1\left(293.19 \pm 27.74,298.21 \pm 31.73,299.60 \pm 29.46,298.11 \pm 29.40 \mathrm{Mg}\right.$ ha- $\left.^{-1}\right)$ and Area $2(254.35 \pm 69.61$, $\left.259.10 \pm 70.05,261.00 \pm 69.43,248.92 \pm 61.78 \mathrm{Mg} . h a-^{-1}\right)$. Besides that, it was noted annual change in stock biomass measurements at intervals of greater than one year due to fluctuations in mortality, recruitment and growth, besides the basal area and abundance of individuals in each area. The highest concentration of individuals occurred in diametric classes DAP $10 \leq \mathrm{DAP} \leq 30 \mathrm{~cm}$, however, it was verified that individuals with DAP $\geq 60 \mathrm{~cm}$ in lower concentration stored biomass equivalent to diametric classes $10 \leq \mathrm{DAP} \leq 30$ $\mathrm{cm}$. We conclude that fluctuations in mortality, recruitment and growth, and abundance and basal area of these sites affected the stock biomass.
\end{abstract}

Keywords: indirect method; diameter distribution; abundance.

1 Engenheiro Florestal, MSc., Instituto Brasileiro do Meio Ambiente e dos Recursos Naturais Renováveis, Av. Tapajós, 2267, Laguinho, CEP 68041-148, Santarém (PA), Brasil. fabioengflorestal@gmail.com

2 Engenheiro Agrônomo, Dr., Professor da Universidade de São Paulo, Av. Centenário, 303, São Dimas, CEP 13416-000, Piracicaba (SP), Brasil. pcamargo@cena.usp.br

3 Engenheiro Agrônomo, Dr., Pesquisador da Empresa Brasileira de Pesquisa Agropecuária, Rua Vera Paz, casa 1, Campus UFOPA - Tapajós, Salé, CEP 68035-110, Santarém (PA), Brasil. raimundo.oliveira-junior@embrapa.br

Recebido para publicação em 11/08/2013 e aceito em 11/07/2017

Ci. Fl., v. 28, n. 3, jul. - set., 2018 


\section{INTRODUÇ̃̃O}

Segundo FAO (ORGANIZAÇÃO DAS NAÇÕES UNIDAS PARA ALIMENTAÇÃO E AGRICULTURA, 2010), floresta é definida como a área que mede mais que meio hectare, com árvores maiores que cinco metros de altura e cobertura de copa superior a 10\%. Adotando este conceito, estima-se que o Brasil possua aproximadamente 516 milhões de hectares de florestas naturais e plantadas, equivalente a $60,7 \%$ do território nacional coberto por florestas, sendo a segunda maior área de florestas conservada do mundo, atrás apenas da Rússia (BRASIL, 2010).

Já que a quantidade de material vegetal presente por unidade de superfície em uma floresta é conceituada como biomassa (ARAÚJO et al., 1999; GUEDES et al., 2001; KEELING; PHILLIPS, 2007), as florestas brasileiras são de fundamental interesse para projetos de conservação referentes ao estoque de biomassa e carbono (SETTE JUNIOR; NAKAJIMA; GEROMINI, 2006; BOINA, 2008).

Os atuais padrões de uso da terra (ARTAXO et al., 2005; KAUFFMAN; HUGHES; HEIDER, 2009) alteram a dinâmica fitossociológica (NASCIMENTO; LAURANCE, 2004; 2006) em florestas tropicais e produzem sérios problemas às gerações presentes e futuras através da queima de biomassa (AIRES; KIRCHHOFF, 2001).

As florestas naturais estão constantemente sob processos de mudanças na estrutura e composição florística, sejam naturais (NASCIMENTO; LAURANCE, 2006; ANDRADE; HIGUCHI, 2009; VIANNA et al., 2010) ou antrópicas (AIRES; KIRCHHOFF, 2001; NASCIMENTO; LAURANCE, 2004), consequentemente alterando os padrões de variação espacial e temporal de biomassa.

As florestas tropicais acumulam um dos maiores estoques de biomassa entre os ecossistemas terrestres, no entanto a sua quantidade exata e os padrões de variação espacial ainda são pouco conhecidos (SARMIENTO PINILLOS; GARAY, 2005).

Guedes et al. (2001), afirmaram que a biomassa é um indicador de produtividade de um sítio e varia com a precipitação, temperatura, latitude e altitude.

Para entender a dinâmica e o estoque de biomassa florestal, e suas relações físico-químicas no ecossistema é necessário avaliar a floresta em seus diversos compartimentos (BOINA, 2008), assim como, as propriedades físico-químicas do solo (CASTILHO, 2004; MALHI et al., 2004), a estrutura populacional e tipologia da vegetação (ANDRADE; HIGUCHI, 2009; VIANNA et al., 2010), além dos fatores climáticos (RICE et al., 2004; KAUFFMAN; HUGHES; HEIDER, 2009). A avaliação e análise desses fatores pode ser feita através de parcelas permanentes de monitoramento (SILVA et al., 2005).

Desta maneira, a quantificação do estoque de biomassa pode ser feita de duas formas: método direto e método indireto. $\mathrm{O}$ método direto consiste na derrubada e pesagem de árvores que ocorre em parcelas fixas, enquanto o método indireto é baseado na estimativa da biomassa individual da árvore através de equações alométricas (HIGUCHI et al., 1998; ANDRADE; HIGUCHI, 2009).

O método direto é trabalhoso, caro e destrutivo e leva em consideração uma área determinada (BROWN et al., 1995; HIGUCHI et al., 1998; GUEDES et al., 2001; NOGUEIRA et al., 2008), e a legislação ambiental estabelece critérios limitadores para supressão florestal, o que dificulta a quantificação de biomassa de áreas extensas.

O método indireto consiste na utilização de dados de inventários florestais e equações alométricas para estimativa de biomassa (HIGUCHI et al., 1998; ANDRADE; HIGUCHI, 2009).

Inicialmente é necessário a construção de um modelo significativamente ajustado, representativo para a população através do método direto (HIGUCHI et al., 1998), contudo, pode-se, em seguida, estimar a biomassa (BROWN et al., 1995; CHAMBERS et al., 2001; MANI; PARTHASARATHY, 2007) de grandes áreas, por meio de uma variável de fácil medição (CHAMBERS et al., 2001; NOGUEIRA et al., 2008), sendo mais rápido e barato em relação ao método direto.

De acordo com Chave et al. (2005), as variáveis mais importantes para construção de modelos alométricos são em ordem decrescente: DAP, densidade da madeira, altura total e tipologia florestal.

As estimativas de biomassa através de equações alométricas bem ajustadas permitem estimativas confiáveis (CHAVE et al., 2005), entretanto, vale lembrar que a biomassa estimada é afetada pela densidade da madeira (FEARNSIDE, 1997; NOGUEIRA; NELSON; FEARNSIDE, 2005), variando dentro do indivíduo, espécie e tipo de floresta, além da forma do tronco e presença de oco na árvore 
(NOGUEIRA; NELSON; FEARNSIDE, 2006).

Estimativas de biomassa em florestas tropicais são essenciais para o entendimento da dinâmica da floresta, estoque de nutrientes, e emissão de gases devido ao desmatamento (BROWN et al., 1995; HIGUCHI et al., 1998; MILLER et al., 2004). Neste sentido, Baker et al. (2004), Feeley et al. (2007), Nogueira et al. (2008) e Mazzei et al. (2010) estimaram estoques de biomassa por meio de equações alométricas.

Considerando que as Unidades de Conservação no oeste do Pará, estão sob constante processo de alterações antrópicas e naturais. O presente trabalho se propõe a conhecer a variabilidade espacial e temporal do estoque de biomassa em duas áreas de floresta ombrófila densa localizada na FLONA Tapajós, além de seu padrão de distribuição, para compreensão da dinâmica e funcionamento desses ecossistemas.

\section{MATERIAIS E MÉTODOS}

\section{Área de estudo}

O presente trabalho foi desenvolvido na Floresta Nacional do Tapajós - FLONA Tapajós, distante aproximadamente $80 \mathrm{~km}$ da cidade de Santarém no sentido sul, nas coordenadas geográficas $03^{\circ} 10^{\prime} 00^{\prime \prime S}$

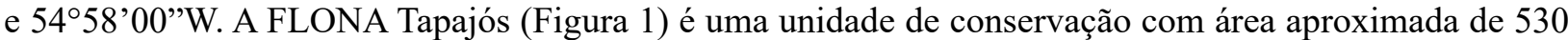
mil hectares, que envolve os municípios de Belterra, Aveiro, Rurópolis e Placas no oeste do Pará (IBAMA, 2004).

A vegetação predominante na área de estudo é classificada como Floresta Ombrófila Densa, caracterizada pela dominância de árvores de grande porte, além de lianas e epífitas (VELOSO; RANGEL FILHO; LIMA, 1991; HERNANDEZ FILHO et al., 1993). Espirito-Santo et al. (2005) através de imagens de satélite classificaram três regiões fitogeográficas distintas na FLONA Tapajós, a saber: porção norte com presença de babaçu bem nítida em campo; porção central com pouca dissimilaridade de espécies entre o alto e baixo platô; e a porção sul rica em palmeiras, exceto babaçu (Orbignya phalerata Mart.).

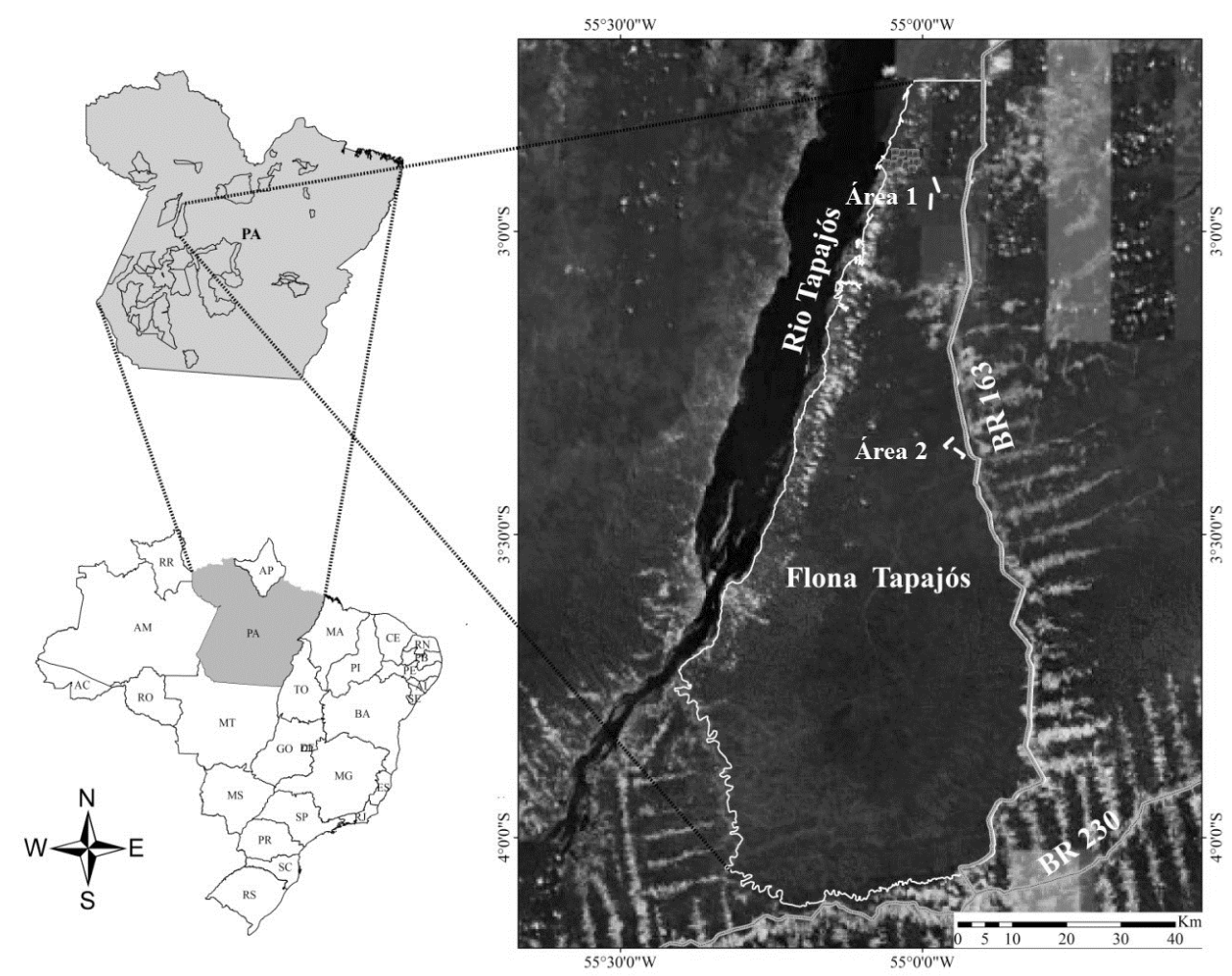

FIGURA 1: Localização das duas áreas de pesquisa (Área 1 e Área 2) (imagem Google Earth/2011, disponível em: http://www.google.com.br/intl/pt-BR/earth/).

FIGURE 1: Locating two research areas (Area 1 e Area 2) (image Google Earth/2011, available in: http://www. google.com.br/intl/pt-BR/earth/). 
O clima na região em estudo apresenta temperatura média de $24,8^{\circ} \mathrm{C}$, umidade relativa média de $90 \%$ e precipitação média anual de $2100 \mathrm{~mm}$, sendo que nos meses de dezembro a maio há maior ocorrência de chuvas e de julho a agosto uma queda pluviométrica brusca, caracterizando-se um período seco no qual a precipitação mensal é inferior a $60 \mathrm{~mm}$ (INSTITUTO NACIONAL DE METEOROLOGIA, 2012). Conforme a classificação de Köppen, enquadra-se no clima "Ami”, caracterizado por apresentar temperatura média do mês mais frio sempre superior a $18^{\circ} \mathrm{C}$ apresentando uma estação seca de pequena duração que é compensada pelos totais elevados de precipitação.

Em relação ao solo predominante na área de pesquisa, ocorre o tipo Latossolo Amarelo Distrófico, caracterizado por diferentes texturas, geralmente profundo, ácido, friável e revestido por florestas densas (ESPÍRITO-SANTO et al., 2005).

\section{Coleta e análise de dados}

Neste trabalho, foi utilizada uma série temporal de dados coletados entre 2007 e 2010, provenientes de doze parcelas permanentes retangulares instaladas em duas áreas no ano 2003 nas proximidades do $\mathrm{km}$ 72, (denominado Área 1) e km 117 (denominado Área 2) da BR 163, que delimita a FLONA Tapajós a oeste.

As parcelas mediram $15 \mathrm{~m}$ x $500 \mathrm{~m}\left(7500 \mathrm{~m}^{2}\right)$, e foram instaladas sistematicamente em área plana não explorada com distância de $500 \mathrm{~m}$ uma da outra, sendo seis parcelas em cada área de estudo, e fazem parte de um Projeto de Pesquisa do Programa LBA (Programa de Grande Escala da Biosfera - Atmosfera na Amazônia), que visa efetuar o monitoramento anual de biomassa florestal.

Todos os indivíduos com Diâmetro a Altura do Peito (DAP) maiores ou iguais a $10 \mathrm{~cm}$ foram medidos, identificados, etiquetados e mapeados no plano cartesiano, dentro de cada parcela para permitir o controle anual das mensurações. O DAP foi medido a $1,30 \mathrm{~m}$ do solo, utilizando-se fitas diamétrica, entre $\neg$ tanto, em casos em que foram constatados sapopemas, nós, cupins, ou alguma alteração na altura do DAP, as medições foram realizadas acima das alterações encontradas.

O modelo alométrico utilizado para o cálculo da estimativa de biomassa foi o trabalhado por Santoni (2004) na FLONA Tapajós, adaptado de Chambers et al. (2001). O modelo original foi construído com dados coletados de 315 indivíduos abatidos em área de estudo de queima de biomassa, nas proximidades de Manaus- AM, visando à quantificação da biomassa individual da parte aérea baseado na relação DAP e biomassa, como demonstrado a seguir:

$$
\text { Biomassa }=\exp ^{\left(0,37+0,333 * \log \mathrm{D}+0,933 *[\log \mathrm{D}]^{2}-0,122 *[\log \mathrm{D}]^{3}\right)}
$$

Sendo: Biomassa $=$ peso seco da biomassa em Kg; D = Diâmetro a Altura do Peito - DAP.

A escolha do modelo foi baseada na literatura, considerando que foram coletados dados dendrométrico somente do DAP para o período analisado, e o mesmo já havia sido testado e comparado a outros modelos (HIGUCHI et al., 1998; CHAVE et al., 2005), em trabalho desenvolvido por Santoni (2004) realizado na FLONA Tapajós, sendo um modelo de simples entrada, com uma variável independente (DAP) de fácil medição, permitindo estimativas similares a outros trabalhos com a mesma temática.

Dentro das áreas de estudo, as parcelas foram medidas anualmente, adotando-se a análise de variância de um fator para medidas repetidas, visando verificar a variabilidade anual e espacial de biomassa entre Área 1 e Área 2.

Para avaliar o grau de relacionamento dos estoques médios anuais de biomassa da Área 1 e Área 2, adotou-se a Correlação de Pearson.

Em relação ao estoque anual de biomassa dentro das áreas de estudo, foi efetuado um teste " $t$ " pareado entre as médias anuais.

Para realização dos testes estatísticos e elaboração de gráficos foram utilizados os softwares Microsoft Office Excel 2007 e SYSTAT 12 para Windows. 


\section{RESULTADOS E DISCUSSÃO}

Os estoques médios de biomassa mostrados na Figura 2 foram diferentes, pois a taxa de mortalidade natural ao longo das medições dos indivíduos com DAP $\geq 60 \mathrm{~cm}$ na Área 2 foi maior que na Área 1, além do mais, o número de indivíduos na Área 1 (Figuras 3 e 4) com DAP $\geq 60 \mathrm{~cm}$ foi maior que na Área 2 (Figuras 5 e 6). Conforme Gale (2000) e Feeley et al. (2007), um dos fatores preponderantes para a diferença pode ser explicado pela formação de clareiras naturais devido às constantes ventanias e queda natural de árvores nas áreas de estudo ao longo do período, entretanto, cada área respondeu diferentemente às intervenções naturais. $\mathrm{O}$ teste de Correlação de Pearson demonstrou que os estoques médios anuais de biomassa das duas áreas possuem baixa correlação $(\mathrm{r}=0,267)$, comprovado pela análise de variância que mostrou diferença significativa entre a Área 1 e a Área 2 (Figura 2).

Chambers et al. (2001) relacionaram a biomassa estimada ao DAP, Mani e Parthasarathy (2007) e Vianna et al. (2010) justificaram a ideia de que a área basal foi um dos fatores que provocou a diferença no estoque de biomassa entre as duas áreas, pois a abundância de indivíduos com DAP $\geq 60 \mathrm{~cm}$ foi maior na Área 1 (Figuras 3 e 4).

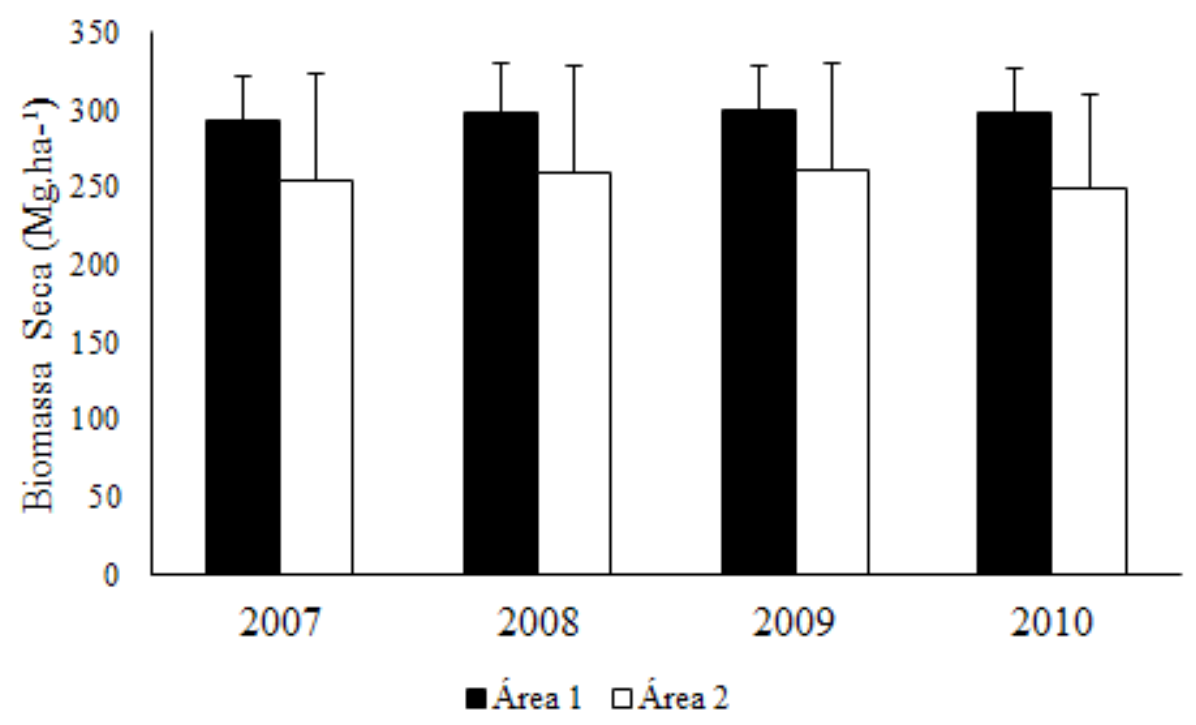

FIGURA 2: Biomassa média anual para os respectivos anos da Área 1 e Área 2 (barras identificam desvio padrão; $\mathrm{r}$ de Pearson $=0,267 ; \mathrm{F}=8,604, \mathrm{p}$ - valor $<0,05$ entre as duas áreas).

FIGURE 2: Biomass for the respective years of Area 1 and Area 2 (bars identify standard deviation; $r$ de Pearson $=$ 0,$267 ; \mathrm{F}=8,604, \mathrm{p}$-value $<0.05$ between the two areas).

Como pode ser observado na Tabela 1, a taxa de mortalidade da Área 2 apresentou aumento acentuado em 2010, sendo que apenas em 2009 foi menor que na Área 1. Apesar da Área 1 possuir aumento na taxa de mortalidade em 2010, a Área 2 perdeu biomassa viva, pois o incremento em biomassa no período para a Área 1 foi de 0,36 Mg.ha.ano- ${ }^{1}$, enquanto na Área 2 foi -0,40 Mg.ha.ano- ${ }^{1}$.

É importante observar que a Área 2 teve aumento na taxa de recrutamento em 2010, possivelmente devido à abertura de clareiras pela alta mortalidade de árvores neste mesmo ano. Segundo Nascimento e Laurance (2006), a entrada de luz na floresta proporciona a germinação de espécies pioneiras e, no caso das clareiras (MALHI et al., 2004; BOINA, 2008) pode reduzir a competição por água, luz e nutrientes, facilitando o ingresso de novos indivíduos nas classes seguintes (GALE; BARFOD, 1999; GALE, 2000; FEELEY et al., 2007).

O incremento em biomassa se manteve praticamente constante, sendo que em 2010 houve uma redução acentuada na Área 2, o que é explicado pela taxa de mortalidade e menor número de indivíduos nas classes com DAP $\geq 60 \mathrm{~cm}$. 
Espirito-Santo et al. (2005) em trabalhos desenvolvidos na FLONA Tapajós, justificaram ainda que a composição florística e abundância das espécies entre Área 1 e Área 2 são diferentes devido à região fitogeográfica em que estão alocadas.

Nogueira, Nelson e Fearnside (2006) reforçaram que irregularidades na forma do fuste e troncos ocos afetaram as estimativas de estoques, pois a biomassa está em função do DAP na equação alométrica usada.

Mani e Parthasarathy (2007) e Vianna et al. (2010) reforçam que a área basal possui uma forte relação com a biomassa, talvez a área ocupada pela população tenha sido preponderante para o maior estoque de biomassa da Área 1, pois nesta área se constatou maior número de indivíduos com DAP $\geq 60$ $\mathrm{cm}$ em relação à Área 2, mas vale lembrar que os estoques de biomassa (Tabela 1) ficaram dentro do valor médio encontrado por Santoni, 2004 (306 Mg.ha- $\left.{ }^{1}\right)$ na FLONA Tapajós para as mesmas áreas de estudo.

Malhi et al. (2004) e Boina (2008) ressaltaram que grande parte da variabilidade no estoque de biomassa ocorre em pequena ou mesoescala espacial, e provavelmente está relacionada com a presença de clareiras e microvariações em propriedades do solo e na topografia, fato que reforça as taxas de mortalidade e formação de clareiras nas duas áreas ao longo do período analisado devido ao vento e queda natural.

Gale e Barfod (1999), Gale (2000) e Feeley et al. (2007) explicaram que as clareiras naturais e o tipo de terreno podem interferir na abundância e mortalidade, além da distribuição das espécies.

TABELA 1: Taxa de Mortalidade (ind.ha- ${ }^{1}$ ), Taxa de Recrutamento (ind.ha- ${ }^{1}$ ) e Biomassa Seca (Mg.ha- ${ }^{1}$ ) no respectivo ano de coleta.

TABLE 1: $\quad$ Mortality Rate (ind.ha- $\left.{ }^{1}\right)$, Fee Recruitment (ind.ha- $\left.{ }^{1}\right)$ e Dry Biomass $\left(\mathrm{Mg}^{\text {.ha- }}{ }^{1}\right)$ in the respective year of collection.

\begin{tabular}{ccccc}
\hline Área & Ano & Mortalidade & Recrutamento & Biomassa Seca \\
\hline & 2007 & - & - & $293,19 \pm 27,74$ \\
& 2008 & $2,31 \% \pm 1,52 \%$ & $1,83 \% \pm 1,01 \%$ & $298,21 \pm 31,73$ \\
Área 1 & & & & \\
& 2009 & $2,33 \% \pm 0,95 \%$ & $2,40 \% \pm 1,08 \%$ & $299,60 \pm 29,46$ \\
& 2010 & $3,05 \% \pm 1,22 \%$ & $1,81 \% \pm 0,71 \%$ & $298,11 \pm 29,40$ \\
& 2007 & - & - & $254,35 \pm 69,61$ \\
& 2008 & $2,99 \% \pm 1,52 \%$ & $2,16 \% \pm 1,51 \%$ & $259,10 \pm 70,05$ \\
Área 2 & & & & \\
& 2009 & $1,89 \% \pm 0,99 \%$ & $0,57 \% \pm 0,56 \%$ & $261,00 \pm 69,43$ \\
& 2010 & $4,74 \% \pm 3,11 \%$ & $1,81 \% \pm 0,94 \%$ & $248,92 \pm 61,78$ \\
\hline
\end{tabular}

O incremento em biomassa seca da Área 1 foi $0,36 \mathrm{Mg} \cdot$ ha.ano- ${ }^{1}$ e na Área 2 foi $-0,40 \mathrm{Mg}$.ha.ano ${ }^{-1}$.

As Figuras 3 a 6 mostram que a estrutura diamétrica do povoamento seguiu o padrão (“j” invertido) usual de florestas tropicais, ou seja, maior concentração de indivíduos nas classes entre $10 \leq \mathrm{DAP} \leq 30 \mathrm{~cm}$, diminuindo com o aumento do diâmetro, indicando a existência de um balanço entre o recrutamento e a mortalidade dos indivíduos (GONÇALVES; SANTOS, 2008). 
O padrão apresentado nas Figuras 3 a 6 indicam que provavelmente a Área 1 e a Área 2 sofreram uma perturbação natural no período estudado (RICE et al., 2004), o que provavelmente reforça o resultado encontrado.

Comparando biomassa e abundância (Figuras 3 e 4; 5 e 6), verificou-se que a Área 2 apresentou maior concentração de indivíduos com $10 \leq \mathrm{DAP} \leq 30 \mathrm{~cm}$ em relação à Área 1, bem como, observou-se que a presença de indivíduos com DAP $\geq 90 \mathrm{~cm}$ na Área 1 foi maior.

A distribuição de biomassa seguiu um padrão equivalente na Área 1 e na Área 2, nas quais, apesar da abundância de indivíduos nas classes com $10 \leq \mathrm{DAP} \leq 30 \mathrm{~cm}$, a grande contribuição no estoque de biomassa ocorreu nas classes com $30 \leq \mathrm{DAP} \leq 60 \mathrm{~cm}$ e com DAP $\geq 90 \mathrm{~cm}$, o que confirma a maior produtividade nos indivíduos com DAP $\geq 30 \mathrm{~cm}$ conforme descrito por Andrade e Higuchi (2009).

Indivíduos com DAP $\geq 90 \mathrm{~cm}$ apresentaram considerável estoque de biomassa, e quando abatidos ou mortos por causas naturais (DELANEY et al., 1998; GALE; BARFOD, 1999) representaram um deficit importante na distribuição e estoque de biomassa viva, pois é possível depreender que possuem ciclo de vida longo e incremento lento de acordo com Nascimento e Laurance (2006), e deste modo a recuperação do estoque pode demorar de dezenas a centenas de anos (LIMA et al., 2007).

$\mathrm{Na}$ análise temporal não houve diferença significativa na Área 1 e na Área 2, contudo, percebeuse através do teste " $t$ " pareado que diferenças podem ocorrer em intervalos de medição maiores, pois na Área 1 houve diferenças significativas entre 2007 e $2009(p=0.0097)$ e 2008 e $2010(p=0.0317)$ e na Área 2 entre 2007 e 2009 ( $p=0.0189$ ) (Figuras 3 a 6). O que se percebe é que essas diferenças ocorreram devido às variações nas taxas de mortalidade, recrutamento e incremento observados nas duas áreas.

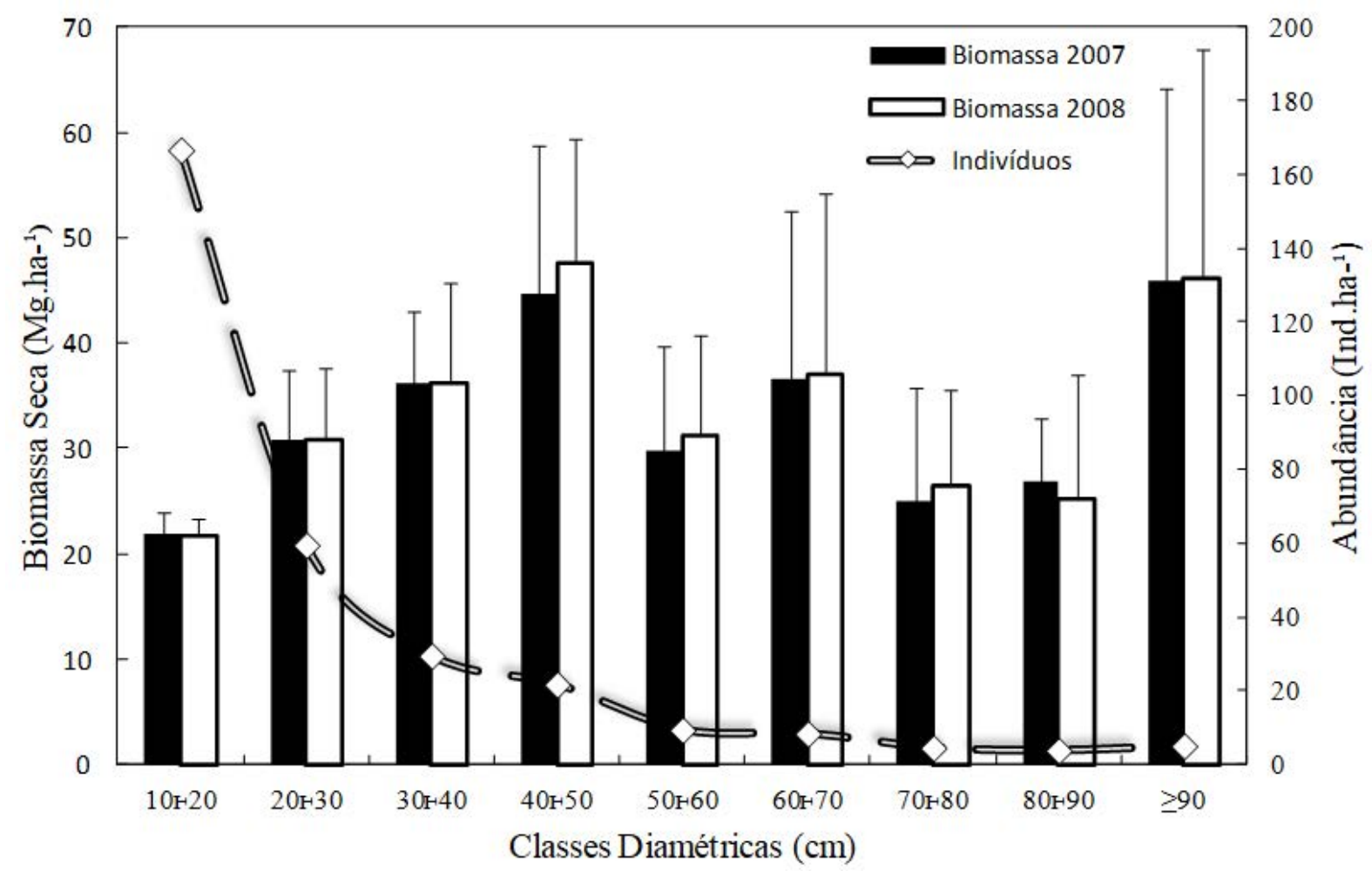

FIGURA 3: Distribuição de biomassa seca e abundância de indivíduos em intervalos de classe de $10 \mathrm{~cm}$ (Área 1: 2007-2008). A abundância está representada pela média anual de indivíduos dos dois anos.

FIGURE 3: Distribution of dry biomass and abundance in class intervals of $10 \mathrm{~cm}$ (Area 1: 2007-2008). Abundance is represented by the average of individuals in both years. 


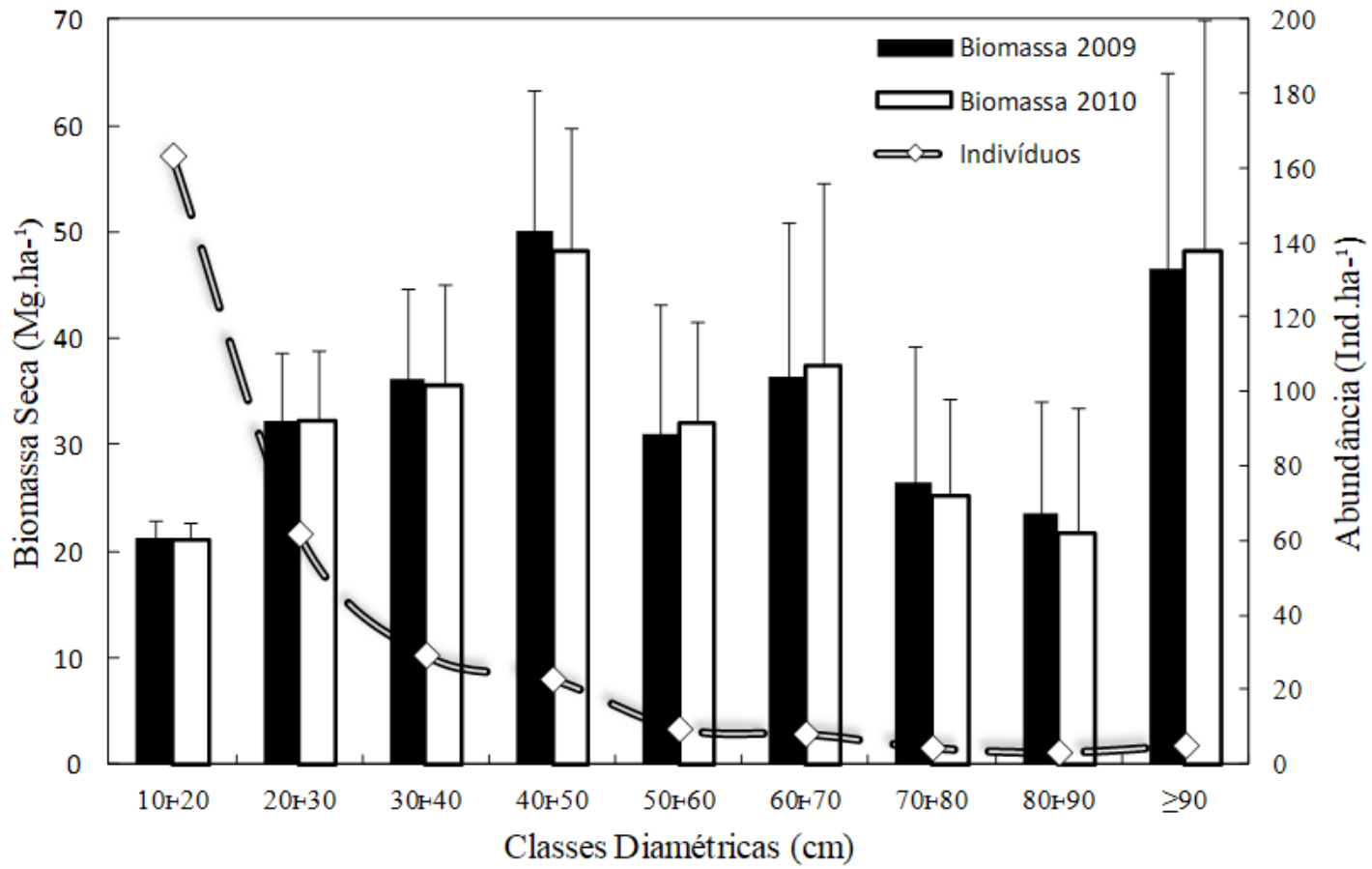

FIGURA 4: Distribuição de biomassa seca e abundância de indivíduos em intervalos de classe de $10 \mathrm{~cm}$ (Área 1: 2009-2010). A abundância está representada pela média anual de indivíduos dos dois anos.

FIGURE 4: Distribution of dry biomass and abundance in class intervals of $10 \mathrm{~cm}$ (Area 1: 2009-2010). Abundance is represented by the average of individuals in both years.

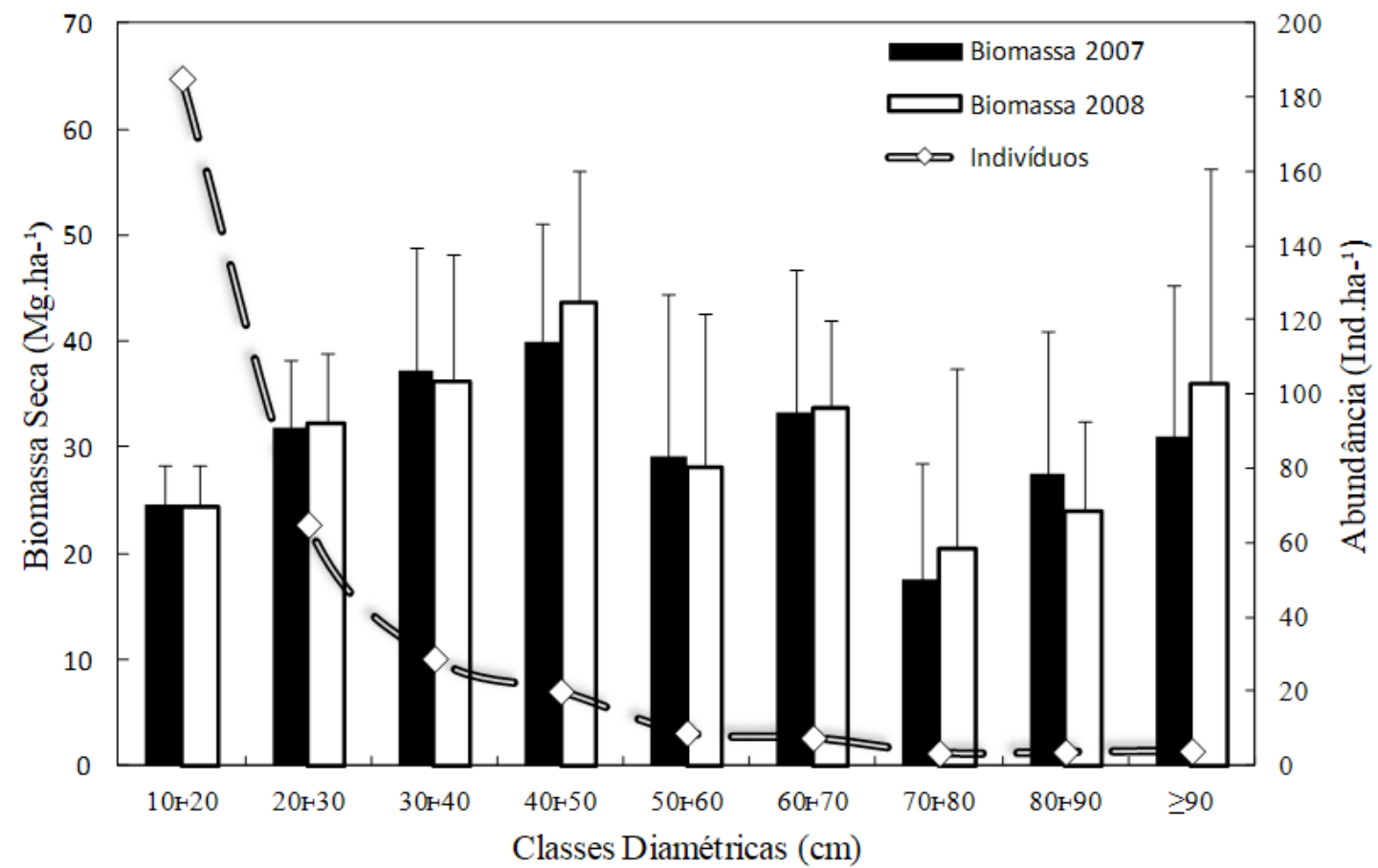

FIGURA 5: Distribuição de biomassa seca e abundância de indivíduos em intervalos de classe de $10 \mathrm{~cm}$ (Área 2: 2007-2008). A abundância está representada pela média anual de indivíduos dos dois anos.

FIGURE 5: Distribution of dry biomass and abundance in class intervals of $10 \mathrm{~cm}$ (Area 2: 2007-2008). Abundance is represented by the average of individuals in both years. 


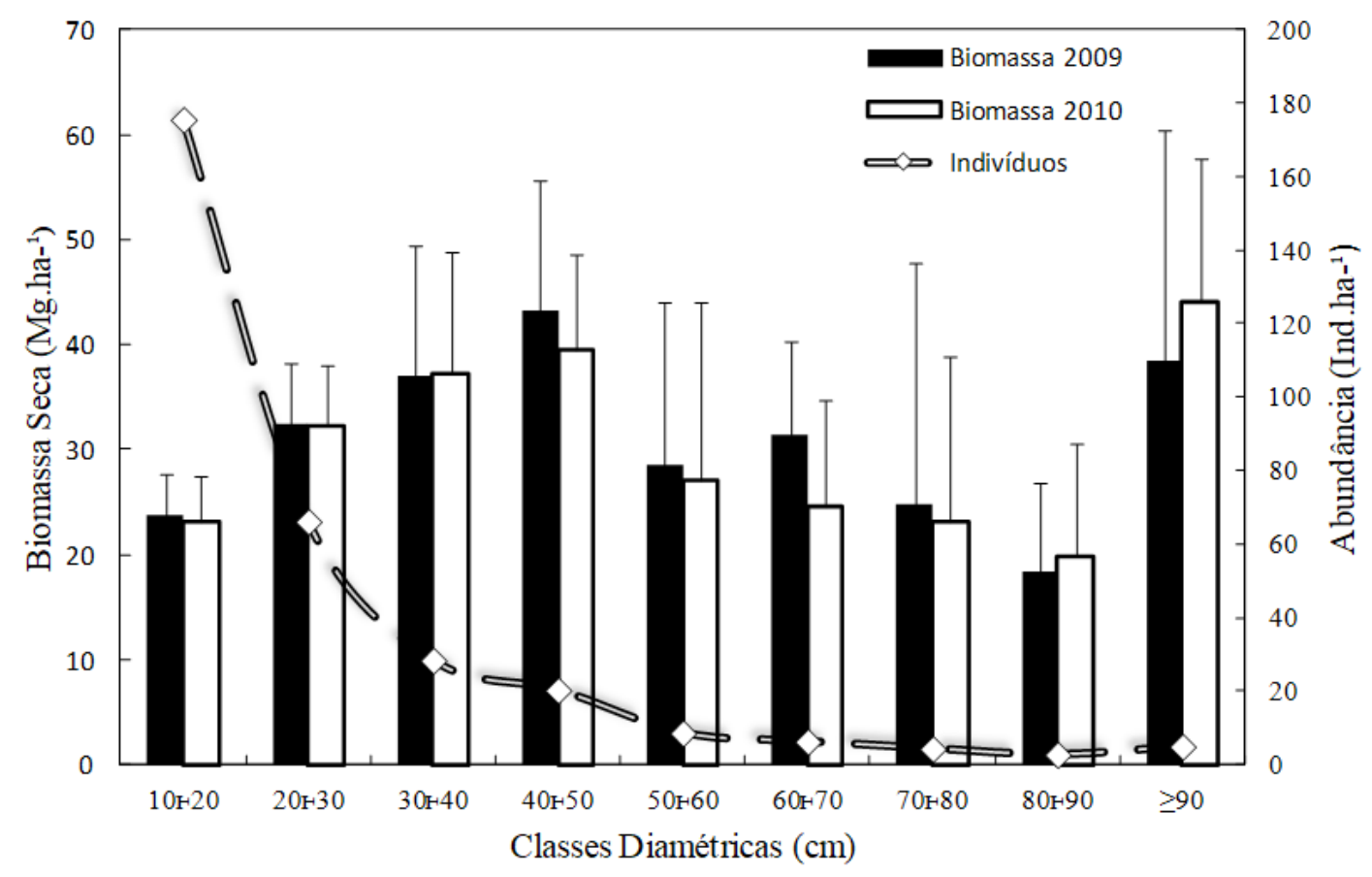

FIGURA 6: Distribuição de biomassa seca e abundância de indivíduos em intervalos de classe de $10 \mathrm{~cm}$ (Área 2: 2009-2010). A abundância está representada pela média anual de indivíduos dos dois anos.

FIGURE 6: Distribution of dry biomass and abundance in class intervals of $10 \mathrm{~cm}$ (Area 2: 2009-2010). Abundance is represented by the average of individuals in both years.

\section{CONCLUSÕES}

A Floresta Ombrófila Densa apresentou variabilidade no estoque de biomassa em escala espacial, sendo que a Área 1 apresentou um estoque de 16,22\% maior que a Área 2, devido provavelmente às taxas de mortalidade e número de indivíduos com $\mathrm{DAP} \geq 60 \mathrm{~cm}$. A variabilidade temporal dentro das áreas de estudo, foi verificada somente em intervalos maiores que um ano de coleta de dados, no entanto, entre a Área 1 e a Área 2 não houve variabilidade significativa, ou seja, as áreas estudadas mantiveram os estoques ao longo dos anos de forma similar.

$\mathrm{O}$ estoque de biomassa sofreu interferência da taxa de mortalidade ao longo das medições, possibilitando um incremento médio anual positivo na Área 1 de $0,36 \mathrm{Mg}$.ha.ano- ${ }^{1}$, enquanto na Área $2 \mathrm{o}$ incremento médio anual teve valor negativo de $-0,40 \mathrm{Mg}$.ha.ano- ${ }^{1}$.

A distribuição de biomassa seguiu um padrão equivalente na Área 1 e na Área 2, sendo os maiores estoques nas classes de $30 \leq \mathrm{DAP} \leq 60 \mathrm{~cm}$ e DAP $\geq 90 \mathrm{~cm}$, demonstrando que apesar do grande número de indivíduos $10 \leq \mathrm{DAP} \leq 30 \mathrm{~cm}$, os indivíduos em classes médias e superiores concentram um grande acúmulo de biomassa.

Estudos posteriores devem ser realizados conciliando a relação tipos de solos e tipologias florestais, além de fatores climáticos. Faz-se necessária também a construção de modelos alométricos ajustados às características fitossociológicas, considerando a estrutura horizontal e vertical da floresta, e com conjuntos de dados representativos da população, a fim de facilitar a tomada de decisão em intervenções na estrutura populacional.

\section{AGRADECIMENTOS}

Ao Programa de Pós-Graduação em Recursos Naturais da Amazônia - PGRNA pela oportunidade de cursar o mestrado. Ao Instituto Brasileiro de Meio Ambiente e dos Recursos Naturais Renováveis IBAMA. Ao Instituto Chico Mendes de Conservação da Biodiversidade - ICMBio. Ao Programa de Grande 
Escala da Biosfera-Atmosfera na Amazônia - LBA pela confiança e inserção no grupo de pesquisa.

\section{REFERÊNCIAS}

AIRES, C. B.; KIRCHHOFF, V. W. J. H. Transporte de monóxido de carbono gerado em queimadas para regiões onde não se queima. Brazilian Journal of Geophysics, São Paulo, v.19, n. 1, p. 61-74, 2001.

ANDRADE, E. A.; HIGUCHI, N. Produtividade de quatro espécies arbóreas de terra firme da Amazônia central. Acta Amazonica, Manaus, v. 39, n. 1, p. 105-112, 2009.

ARAÚJO, T. M. et al. Comparison of formulae for biomass content determination in a tropical rain forest site in the state of Pará, Brazil. Forest Ecology and Management, Amsterdam, v. 117, p. 43-52, 1999.

ARTAXO, P. et al. Química atmosférica na Amazônia: a floresta e as emissões de queimadas controlando a composição da atmosfera amazônica. Acta Amazonica, Manaus, v. 35, n. 2, p. 185-196, 2005.

BAKER, T. R. et al. Increasing biomass in Amazonian forest plots. Philosophical Transactions, Londres, v. 359, p. 353-365, 2004.

BOINA, A. Quantificação de estoques de biomassa e de carbono em floresta estacional semidecidual, Vale do Rio Doce, Minas Gerais. 2008. 89 f. Dissertação (Mestrado) - Universidade Federal de Viçosa, Viçosa, MG. 2008.

BRASIL. Ministério do Meio Ambiente. Florestas do Brasil: em resumo. Brasília: MMA, 2010. 152 p. BROWN, I. F. et al. Uncertainty in the biomass of Amazonian forests: an example from Rondônia; Brazil. Forest Ecology and Management, Amsterdam, v. 75, p. 175-189, 1995.

CASTILHO, C. V. Variação espacial e temporal da biomassa arbórea viva em $64 \mathbf{~ k m}^{2}$ de floresta de terra-firme na amazônia central. 2004. 87 f. Tese (Doutorado) - Instituto Nacional de Pesquisas da Amazônia, Manaus, 2004.

CHAMBERS, J. Q. et al. Tree damage, allometric relationships and above-ground in central Amazon forest. Forest Ecology and Managements, Amsterdam, v. 152, p. 73-84, 2001.

CHAVE, J. et al. Tree allometry and improved estimation of carbon stocks and balance in tropical forests. Oecologia, São Paulo, v. 145, n. 87, p. 87-99, 2005.

DELANEY, M.; BROWN, S.; LUGO, A. E.; TORRES-LEZAMA, A.; QUINTERO, N. B. The quantity and turnover of dead wood in permanent forest plots in six life zones of Venezuela. Biotropica, Flórida, p. 30:2-11, 1998.

ESPIRITO-SANTO, F. D. B. et al. Análise da composição florística e fitossociológica da Floresta Nacional do Tapajós com o apoio geográfico de imagens de satélites. Acta Amazonica, Manaus, v. 35, n. 2, p. 155-173, 2005.

FEARNSIDE, P. M. Wood density for estimating forest biomass in Brazilian Amazonia. Forest Ecology and Management, Amsterdam, v. 90, p. 59-89, 1997.

FEELEY, K. J. et al. The role of gap phase processes in the biomass dynamics of tropical forests. Biological Sciences, Maringá, v. 274, n. 1627, p. 2857-2864, 2007.

GALE, N., BARFOD, A.S. Canopy tree mode of death in a western Ecuadorian rain forest. Journal of Tropical Ecology, Cambridge, p. 15:415-436, 1999.

GALE, N. The relationship between canopy gaps and topography in a western Ecuadorian rain forest. Biotropica, Flórida, p. 32:653-661, 2000.

GONÇALVES, F. G.; SANTOS, J. R. Composição florística e estrutura de uma unidade de manejo florestal sustentável na Floresta Nacional do Tapajós, Pará. Acta Amazonica, Manaus, v. 38, n. 2, p. 229-244, 2008. GUEDES, B. et al. Estudo da biomassa florestal numa floresta aberta de miombo no distrito de Báruè, Manica. In: RELATÓRIO das actividades de julho. [s. 1.: s. n.], 2001. v. 2. p. 1-15.

HERNANDEZ FILHO, P. et al. Relatório final do projeto de inventário florestal na Floresta Nacional do Tapajós. São José dos Campos: INPE, 1993. 126 p.

HIGUCHI, N. et al. Biomassa da parte área da vegetação da floresta tropical úmida de terra-firme da Amazônia brasileira. Acta Amazonica, Manaus, v. 28, n. 2, p. 153-166, 1998.

INSTITUTO NACIONAL DE METEOROLOGIA. Normais climatológicas. Brasília: INMET, 2012. Disponível em: <www.inmet.gov.br>. Acesso em: 08 mar. 2012.

LIMA, A. J. N. et al. Análise da estrutura e do estoque de fitomassa de uma floresta secundária da 
região de Manaus AM, dez anos após corte raso seguido de fogo. Acta Amazonica, Manaus, v. 37, n. 1, p. 49-54, 2007.

IBAMA. Floresta Nacional do Tapajós: plano de manejo. 2004. Disponível em: $<$ http://www4.icmbio.gov.br/flona_tapajos/>. Acesso em: 29 dez. 2010.

KAUFFMAN, J. B.; HUGHES, R. F.; HEIDER, C. Carbon pool and biomass dynamics associated with deforestation, land use, and agricultural abandonment in the neotropics. Ecological Applications, Washington, v. 19, p. 1211-1222, 2009.

KEELING, H. C.; PHILLIPS, O. L. The global relationship between Forest productivity and biomass. Global Ecology and Biogeography, Malden, v. 16, p. 618-631, 2007.

MALHI, Y. et al. The above-ground coarse wood productivity of 104 neotropical forest plots. Global Change Biology, Malden, v. 10, p. 563-591, 2004.

MANI, S.; PARTHASARATHY, N. Above-ground biomass estimation in ten tropical dry evergreen forest sites of peninsular India. Biomass \& Bioenergy, Amsterdam, v. 31, p. 284-290, 2007.

MAZZEI, L. et al. Above-ground biomass dynamics after reduced-impact logging in the eastern Amazon. Forest Ecology and Management, Amsterdam, v. 259, p. 367-373, 2010.

MILLER, S. D. et al. Biometric and micrometeorological measurements of tropical forest carbon balance. Ecological Applications, São Paulo, v. 14, n. 4, p. 114-126, 2004.

NASCIMENTO, H. E. M.; LAURANCE, W. F. Biomass dynamics in Amazonian forest fragments. Ecological Applications, São Paulo, v. 14, n. 4, p. 127-138, 2004.

NASCIMENTO, H. E. M.; LAURANCE, W. F. Efeito de área e de borda sobre a estrutura florestal em fragmentos de floresta de terra-firme após 13-17 anos de isolamento. Acta Amazonica, Manaus, v. 36, n. 2, p. 183-192, 2006.

NOGUEIRA, E. M. et al. Estimates of forest biomass in the Brazilian Amazon: new allometric equations and adjustments to biomass from wood-volume inventories. Forest Ecology and Management, Amsterdam, v. 256, p. 1853-1867, 2008.

NOGUEIRA, E. M.; NELSON, B. W.; FEARNSIDE, P. M. Volume and biomass of trees in central Amazonia: influence of irregularly shaped and hollow trunks. Forest Ecology and Management, Amsterdam, v. 227, p. 14-21, 2006.

NOGUEIRA, E. M.; NELSON, B. W.; FEARNSIDE, P. M. Wood density in dense forest in central Amazonia, Brazil. Forest Ecology and Management, Amsterdam, v. 208, p. 261-286, 2005.

ORGANIZAÇÃO DAS NAÇÕES UNIDAS PARA ALIMENTAÇÃO E AGRICULTURA. Global Forest Resources Assesment Update 2005: terms and definitions. 2010. Disponível em: <www. fao.org/forestry/media/7797/1/0>. Acesso em: 15 maio 2010.

RICE, A. H. et al. Carbon balance and vegetation dynamics in an old-growth Amazonian forest. Ecological Applications, São Paulo, v. 14, n. 4, p. 55-71, 2004.

SANTONI, G. Landscape-Scale spatial variability of carbon in live and dead biomass in the Tapajós National Forest, Brazil. 2004. 56 f. Tese (Doutorado) - Harvard University, Cambridge, 2004.

SARMIENTO, G.; PINILLOS, M.; GARAY, I. Biomass variability in tropical American lowland rainforests. Ecotropicos, Mérida, v. 18, n. 1, p. 1-20, 2005.

SETTE JÚNIOR, C. R.; NAKAJIMA, N. Y.; GEROMINI, M. P. Captura de carbono orgânico em povoamentos de Pinus taeda L. na região de rio negrinho, SC. Floresta, Curitiba, v. 36, n. 1, p. 36, 2006.

SILVA, J. N. M. et al. Diretrizes para instalação e medição de parcelas permanentes em florestas naturais da Amazônia Brasileira. [s. 1.]: Embrapa; ITTO, 2005. 68 p.

VELOSO, H. M.; RANGEL FILHO, A. L. R.; LIMA, J. C. A. Classificação da vegetação brasileira, adaptada a um sistema universal. Rio de Janeiro: Ministério da Economia, Fazenda e Planejamento, 1991. $124 \mathrm{p}$.

VIANNA, A. L. M. et al. Tamanho ótimo de parcela para estimativa de estoque de carbono da parte aérea lenhosa de fitofisionomias florestais na Amazônia oriental. Floresta, Curitiba, v. 40, n. 2, p. 447-456, 2010. 